\title{
Comparing a Linguistic and a Stochastic Tagger
}

\author{
Christer Samuelsson \\ Lucent Technologies \\ Bell Laboratories \\ 600 . Nountain Ave, Room 2D-339 \\ Murray Hill, NJ 07974, USA \\ christeroresearch. bell-labs.com \\ Atro Voutilainen \\ Research Unit for Multilingual Language Technology \\ P.O. Box 4 \\ FIN-00014 University of Helsinki \\ Finland \\ Atro. Voutilainen@Helsinki.FI
}

\begin{abstract}
Concerning different approaches to automatic PoS tagging: EngCG-2, a constraintbased morphological tagger, is compared in a double-blind test with a state-of-the-art statistical tagger on a common disambiguation task using a common tag set. The experiments show that for the same amount of remaining ambiguity, the error rate of the statistical tagger is one order of magnitude greater than that of the rule-based one. The two related issues of priming effects compromising the results and disagreement between human annotators are also addressed.
\end{abstract}

\section{Introduction}

There are currently two main methods for automatic part-of-speech tagging. The prevailing one uses essentially statistical language models automatically derived from usually hand-annotated corpora. These corpus-based models can be represented e.g. as collocational matrices (Garside et al. (eds.) 1987: Church 1988), Hidden Markov models (cf. Cutting et al. 1992). local rules (e.g. Hindle 1989) and neural networks (e.g. Schmid 1994). Taggers using these statistical language models are generally reported to assign the correct and unique tag to $95-97 \%$ of words in running text. using tag sets ranging from some dozens to about 130 tags.

The less popular approach is based on hand-coded linguistic rules. Pioneering work was done in the 1960 s (e.g. Greene and Rubin 1971). Recently, new interest in the linguistic approach has been shown e.g. in the work of (Karlsson 1990: Voutilainen et al. 1992; Oflazer and Kuruöz 1994: Chanod and Tapanainen 1995: Karlsson et al. (eds.) 1995; Voutilainen 1995). The first serious linguistic competitor to data-driven statistical taggers is the English Constraint Grammar parser. EngCG (cf. Voutilainen et al. 1992; hartsson et al. (eds.) 1995). The tagger consists of the following sequentially applied modules:
1. Tokenisation

2. Morphological analysis

(a) Lexical component

(b) Rule-based guesser for unknown words

3. Resolution of morphological ambiguities

The ragger uses a two-level morphological analyser with a large lexicon and a morphological description that introduces about 180 different ambiguity-forming morphological analyses, as a result of which each word gets 1.7-2.2 different analyses on an average. Morphological analyses are assigned to unknown words with an accurate rulebased 'guesser'. The morphological disambiguator uses constraint rules that discard illegitimate morphological analyses on the basis of local or global context conditions. The rules can be grouped as ordered subgrammars: e.g. heuristic subgrammar 2 can be applied for resolving ambiguities left pending by the more 'careful' subgrammar 1 .

Older versions of EngCG (using about 1,150 constraints) are reported (Voutilainen et al. 1992; Voutilainen and Heikkilä 1994; Tapanainen and Voutilainen 1994; Voutilainen 1995) to assign a correct analysis to about $99.7 \%$ of all words while each word in the output retains 1.04-1.09 alternative analyses on an average, i.e. some of the ambiguities remain unresolved.

These results have been seriously questioned. One doubt concerns the notion "correct analysis". For example Church (1992) argues that linguists who manually perform the tagging task using the doubleblind method disagree about the correct analysis in at least $3 \%$ of all words even after they have negotiated about the initial disagreements. If this were the case, reporting accuracies above this $9 \pi \%$ upper bound' would make no sense.

However, Voutilainen and Järvinen (1995) empirically show that an interjudge agreement virtually of $100 \%$ is possible. at least with the EngCG tag set if not with the original Brown Corpus tag set. This consistent applicability of the EngCG tag set is explained by characterising it as grammatically rather than semantically motivated. 
Another main reservation about the EngCG figures is the suspicion that, perhaps partly due to the somewhat underspecific nature of the EngCG tag set, it must be so easy to disambiguate that also a statistical tagger using the EngCG tags would reach at least as good results. This argument will be examined in this paper. It will be empirically shown (i) that the EngCG tag set is about as difficult for a probabilistic tagger as more generally used tag sets and (ii) that the EngCG disambiguator has a clearly smaller error rate than the probabilistic tagger when a similar (small) amount of ambiguity is permitted in the output.

A state-of-the-art statistical tagger is trained on a corpus of over 350,000 words hand-annotated with EngCG tags. then both taggers (a new version known as EngCG-2 ${ }^{1}$ with 3,600 constraints as five subgrammars ${ }^{2}$, and a statistical tagger) are applied to the same held-out benchmark corpus of $5 \overline{5}, 000$ words, and their performances are compared. The results disconfirm the suspected 'easiness' of the EngCG tag set: the statistical tagger's performance figures are no better than is the case with better known tag sets.

Two caveats are in order. What we are not addressing in this paper is the work load required for making a rule-based or a data-driven tagger. The rules in EngCG certainly took a considerable effort to write, and though at the present state of knowledge rules could be written and tested with less effort, it may well be the case that a tagger with an accuracy of $95-97 \%$ can be produced with less effort by using data-driven techniques. ${ }^{3}$

Another caveat is that EngCG alone does not resolve all ambiguities, so it cannot be compared to a typical statistical tagger if full disambiguation is required. However, Voutilainen (1995) has shown that EngCG combined with a syntactic parser produces morphologically unambiguous output with an accuracy of $99.3 \%$, a figure clearly better than that of the statistical tagger in the experiments below (however, the test data was not the same).

Before examining the statistical tagger. two practical points are addressed: the annotation of the corpora used, and the modification of the EngCG tag set for use in a statistical tagger.

\footnotetext{
${ }^{1}$ An online version of $\mathrm{EngCC}-2$ can be found at http://www.ling.helsinki.fi/" avoutila/engcg-2.html.

${ }^{2}$ The first three subgrammars are generally highly reliable and almost all of the total grammar development time was spent on them: the last two contain rather rough heuristic constraints.

${ }^{3}$ However, for an interesting experiment suggesting otherwise, see (Chanod and Tapanainen 1995).
}

\section{Preparation of Corpus Resources}

\subsection{Annotation of training corpus}

The stochastic tagger was trained on a sample of 357,000 words from the Brown University Corpus of Present-Day English (Francis and Kučera 1982) that was annotated using the EngCG tags. The corpus was first analysed with the EngCG lexical analyser, and then it was fully disambiguated and, when necessary, corrected by a human expert. This annotation took place a few years ago. Since then, it has been used in the development of new EngCG constraints (the present version, EngCG-2, contains about 3,600 constraints): new constraints were applied to the training corpus, and whenever a reading marked as correct was discarded, either the analysis in the corpus, or the constraint itself, was corrected. In this way, the tagging quality of the corpus was continuously improved.

\subsection{Annotation of benchmark corpus}

Our comparisons use a held-out benchmark corpus of about 55,000 words of journalistic, scientific and manual texts, i.e., no training effects are expected for either system. The benchmark corpus was annotated by first applying the preprocessor and morphological analyser, but not the morphological disambiguator, to the text. This morphologically ambiguous text was then independently and fully disambiguated by two experts whose task was also to detect any errors potentially produced by the previously applied components. They worked independently, consulting written documentation of the tag set when necessary. Then these manually disambiguated versions were automatically compared with each other. At this stage, about $99.3 \%$ of all analyses were identical. When the differences were collectively examined, virtually all were agreed to be due to clerical mistakes. Only in the analysis of 21 words, different (meaning-level) interpretations persisted, and even here both judges agreed the ambiguity to be genuine. One of these two corpus versions was modified to represent the consensus. and this 'consensus corpus' was used as a benchmark in the evaluations.

As explained in Voutilainen and Järvinen (1995). this high agreement rate is due to two main factors. Firstly, distinctions based on some kind of vague semantics are avoided. which is not always case with better known tag sets. Secondly, the adopted analysis of most of the constructions where humans tend to be uncertain is documented as a collection of tag application principles in the form of a grammarian s manual (for further details, $c f$. Voutilainen and Järvinen 1995).

The corpus-annotation procedure allows us to perform a text-book statistical hypothesis test. Let the null hypothesis be that any two human eva!uators will necessarily disagree in at least $3 \%$ of 
the cases. Under this assumption, the probability of an observed disagreement of less than $2.88 \%$ is less than $5 \%$. This can be seen as follows: For the relative frequency of disagreement, $f_{n}$, we have that $f_{n}$ is approximately $\sim N\left(p, \sqrt{\frac{p(1-p)}{n}}\right)$, where $p$ is the actual disagreement probability and $n$ is the number of trials, i.e.. the corpus size. This means that $P\left(\left(\frac{f_{n}-p}{\sqrt{p(1-p)}} \sqrt{n} \leq x\right) \approx \Phi(x)\right.$ where $\Phi$ is the standard normal distribution function. This in turn means that

$$
P\left(f_{n} \leq p+x \sqrt{\frac{p(1-p)}{n}}\right) \approx \Phi(x)
$$

Here $n$ is 55,000 and $\Phi(-1.645)=0.05$. Under the null hypothesis, $p$ is at least $3 \%$ and thus:

$$
\begin{gathered}
P\left(f_{n} \leq 0.03-1.645 \sqrt{\frac{0.03 \cdot 0.97}{55.000}}\right)= \\
=P\left(f_{n} \leq 0.0288\right) \leq 0.05
\end{gathered}
$$

We can thus discard the null hypothesis at significance level $5 \%$ if the observed disagreement is less than $2.88 \%$. It was in fact $0.7 \%$ before error correction, and virtually zero $\left(\frac{21}{55,000}\right)$ after negotiation. This means that we can actually discard the hypotheses that the human evaluators in average disagree in at least $0.8 \%$ of the cases before error correction, and in at least $0.1 \%$ of the cases after negotiations, at significance level $5 \%$.

\subsection{Tag set conversion}

The EngCG morphological analyser's output formally differs from most tagged corpora; consider the following j-ways ambiguous analysis of "walk":

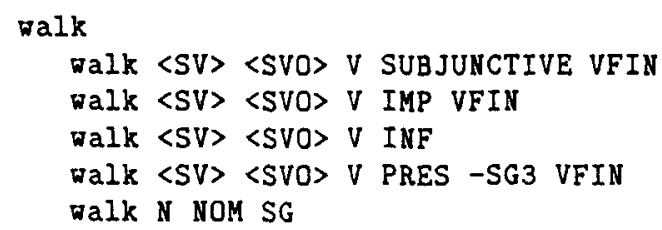

Statistical taggers usually employ single tags to indicate analyses (e.g. "NN" for "N NOM SG"). Therefore a simple conversion program was made for producing the following kind of output, where each reading is represented as a single tag:

\section{walk V-SUBJUNCTIVE V-IMP V-INF $V$-PRES-BASE N-NOM-SG}

The conversion program reduces the multipart EngCG tags into a set of 80 word tags and 17 punctuation tags (see Appendix) that retain the central linguistic characteristics of the original EngCG tag set.
A reduced version of the benchmark corpus was prepared with this conversion program for the statistical tagger's use. Also EngCG's output was converted into this format to enable direct comparison with the statistical tagger.

\section{The Statistical Tagger}

The statistical tagger used in the experiments is a classical trigram-based HMM decoder of the kind described in e.g. (Church 1988), (DeRose 1988) and numerous other articles. Following conventional notation, e.g. (Rabiner 1989, pp. 272-274) and (Krenn and Samuelsson 1996, pp. 42-46), the tagger recursively calculates the $\alpha, 3, \gamma$ and $\delta$ variables for each word string position $t=1, \ldots, T$ and each possible state $^{4} s_{i}: i=1, \ldots, n$ :

$$
\begin{aligned}
\alpha_{t}(i) & =P\left(\mathbf{W}_{\leq t} ; S_{t}=s_{i}\right) \\
\beta_{t}(i) & =P\left(\mathbf{W}_{>t} \mid S_{t}=s_{i}\right) \\
\gamma_{t}(i) & =P\left(S_{t}=s_{i} \mid \mathbf{W}\right)=\frac{P\left(\mathbf{W} ; S_{t}=s_{i}\right)}{P(\mathbf{W})}= \\
& =\frac{\alpha_{t}(i) \cdot 3_{t}(i)}{\sum_{i=1}^{n} \alpha_{t}(i) \cdot 3_{t}(i)} \\
\delta_{t}(i) & =\operatorname{Sax}_{\leq t-1} P\left(\mathbf{S}_{\leq t-1}, S_{t}=s_{i} ; \mathbf{W}_{\leq t}\right)
\end{aligned}
$$

Here

$$
\begin{aligned}
\mathbf{W} & =W_{1}=w_{k_{1}}, \ldots, W_{T}=w_{k_{T}} \\
\mathbf{W}_{\leq t} & =W_{1}=w_{k_{1}}, \ldots, W_{t}=w_{k_{t}} \\
\mathbf{W}_{>t} & =W_{t+1}=w_{k_{t}+1}, \ldots, W_{T}=w_{k_{T}} \\
\mathbf{S}_{\leq t} & =S_{1}=s_{i_{1}}, \ldots, S_{t}=s_{i_{t}}
\end{aligned}
$$

where $S_{t}=s_{i}$ is the event of the $t$ th word being emitted from state $s_{i}$ and $W_{t}=w_{k_{t}}$ is the event of the th word being the particular word $w_{k_{t}}$ that was actually observed in the word string.

Note that for $t=1, \ldots, T-1 ; i, j=1, \ldots, n$

$$
\begin{aligned}
\alpha_{t+1}(j) & =\left[\sum_{i=1}^{n} \alpha_{t}(i) \cdot p_{i j}\right] \cdot a_{j k_{t+1}} \\
3_{t}(i) & =\sum_{j=1}^{n} 3_{t+1}(j) \cdot p_{i j} \cdot a_{j k_{t+1}} \\
\delta_{t+1}(j) & =\left[\max _{i} \delta_{t}(i) \cdot p_{i j}\right] \cdot a_{j k_{t+1}}
\end{aligned}
$$

where $p_{i j}=P\left(S_{t+1}=s_{j} \mid S_{t}=s_{i}\right)$ are the transition probabilities, encoding the tag $\mathrm{N}$-gram probabilities, and

$$
\begin{aligned}
& a_{j k}= \\
& \quad=P\left(W_{t}=u_{k} \mid S_{t}=s_{j}\right)=P\left(U_{t}=u_{k} \mid x_{t}=x_{j}\right)
\end{aligned}
$$

${ }^{4}$ The $\mathrm{V}$ - $l$ th-order H.M.M corresponding to an N-gram tagger is encoded as a first-order H.MM, where each state corresponds to a sequence of $V-1$ tags, i.e.. for a trigram tagger, each state corresponds to a tag pair. 
are the lexical probabilities. Here $X_{t}$ is the random variable of assigning a tag to the $t$ th word and $x_{j}$ is the last tag of the tag sequence encoded as state $s_{j}$. Note that $s_{i} \neq s_{j}$ need not imply $x_{i} \neq x_{j}$.

More precisely, the tagger employs the converse lexical probabilities

$$
a_{j k}^{\prime}=\frac{P\left(X_{t}=x_{j} \mid W_{t}=w_{k}\right)}{P\left(X_{t}=x_{j}\right)}=\frac{a_{j k}}{P\left(W_{t}=w_{k}\right)}
$$

This results in slight variants $\alpha^{\prime}, \beta^{\prime}, \gamma^{\prime}$ and $\delta^{\prime}$ of the original quantities:

$$
\begin{aligned}
& \frac{\alpha_{t}(i)}{\alpha_{t}^{\prime}(i)}=\frac{\delta_{t}(i)}{\delta_{t}^{\prime}(i)}=\prod_{u=1}^{t} P\left(W_{u}=w_{k_{u}}\right) \\
& \frac{\beta_{t}(i)}{\beta_{t}^{\prime}(i)}=\prod_{u=t+1}^{T} P\left(W_{u}=w_{k_{u}}\right)
\end{aligned}
$$

and thus $\forall i, t$

$$
\begin{aligned}
\gamma_{t}^{\prime}(i) & =\frac{\alpha_{t}^{\prime}(i) \cdot \beta_{t}^{\prime}(i)}{\sum_{i=1}^{n} \alpha_{t}^{\prime}(i) \cdot \beta_{t}^{\prime}(i)}= \\
& =\frac{\alpha_{t}(i) \cdot \beta_{t}(i)}{\sum_{i=1}^{n} \alpha_{t}(i) \cdot \beta_{t}(i)}=\gamma_{t}(i)
\end{aligned}
$$

and $\forall t$

$$
\underset{1 \leq i \leq n}{\operatorname{argmax}} \delta_{t}^{\prime}(i)=\underset{1 \leq i \leq n}{\operatorname{argmax}} \delta_{t}(i)
$$

The rationale behind this is to facilitate estimating the model parameters from sparse data. In more detail, it is easy to estimate $P(t a g \mid$ word $)$ for a previously unseen word by backing off to statistics derived from words that end with the same sequence of letters (or based on other surface cues), whereas directly estimating $P$ (word $\mid t a g)$ is more difficult. This is particularly useful for languages with a rich inflectional and derivational morphology, but also for English: for example, the suffix "-tion" is a strong indicator that the word in question is a noun; the suffix "-able" that it is an adjective.

More technically, the lexicon is organised as a reverse-suffix tree, and smoothing the probability estimates is accomplished by blending the distribution at the current node of the tree with that of higherlevel nodes, corresponding to (shorter) suffixes of the current word (suffix). The scheme also incorporates probability distributions for the set of capitalized words, the set of all-caps words and the set of infrequent words, all of which are used to improve the estimates for unknown words. Employing a small amount of back-off smoothing also for the known words is useful to reduce lexical tag omissions. Empirically, looking two branching points up the tree for known words, and all the way up to the root for unknown words, proved optimal. The method for blending the distributions applies equally well to smoothing the transition probabilities $p_{i j}$, i.e., the tag N-gram probabilities, and both the scheme and its application to these two tasks are described in detail in (Samuelsson 1996), where it was also shown to compare favourably to (deleted) interpolation, see (Jelinek and Mercer 1980), even when the back-off weights of the latter were optimal.

The $\delta$ variables enable finding the most probable state sequence under the HMM, from which the most likely assignment of tags to words can be directly established. This is the normal modus operandi of an HMM decoder. Using the $\gamma$ variables, we can calculate the probability of being in state $s_{i}$ at string position $t$, and thus having emitted $w_{k_{t}}$ from this state, conditional on the entire word string. By summing over all states that would assign the same tag to this word, the individual probability of each tag being assigned to any particular input word, conditional on the entire word string, can be calculated:

$$
\begin{aligned}
& P\left(X_{t}=x_{i} \mid \mathbf{W}\right)= \\
& \quad=\sum_{s_{j}: x_{j}=x_{i}} P\left(S_{t}=s_{j} \mid \mathbf{W}\right)=\sum_{s_{j}: x_{j}=x_{i}} \gamma_{t}(j)
\end{aligned}
$$

This allows retaining multiple tags for each word by simply discarding only low-probability tags; those whose probabilities are below some threshold value. Of course, the most probable tag is never discarded, even if its probability happens to be less than the threshold value. By varying the threshold, we can perform a recall-precision, or error-rate-ambiguity, tradeoff. A similar strategy is adopted in (de Marcken 1990).

\section{Experiments}

The statistical tagger was trained on 357,000 words from the Brown corpus (Francis and Kučera 1982), reannotated using the EngCG annotation scheme (see above). In a first set of experiments, a 35,000 word subset of this corpus was set aside and used to evaluate the tagger's performance when trained on successively larger portions of the remaining 322,000 words. The learning curve, showing the error rate after full disambiguation as a function of the amount of training data used, see Figure 1, has levelled off at 322,000 words, indicating that little is to be gained from further training. We also note that the $a b-$ solute value of the error rate is $3.51 \%$ - a typical state-of-the-art figure. Here, previously unseen words contribute $1.08 \%$ to the total error rate, while the contribution from lexical tag omissions is $0.08 \%$. $95 \%$ confidence intervals for the error rates would range from $\pm 0.30 \%$ for 30,000 words to $\pm 0.20 \%$ at 322,000 words.

The tagger was then trained on the entire set of 357,000 words and confronted with the separate 55,000 -word benchmark corpus, and run both in full 


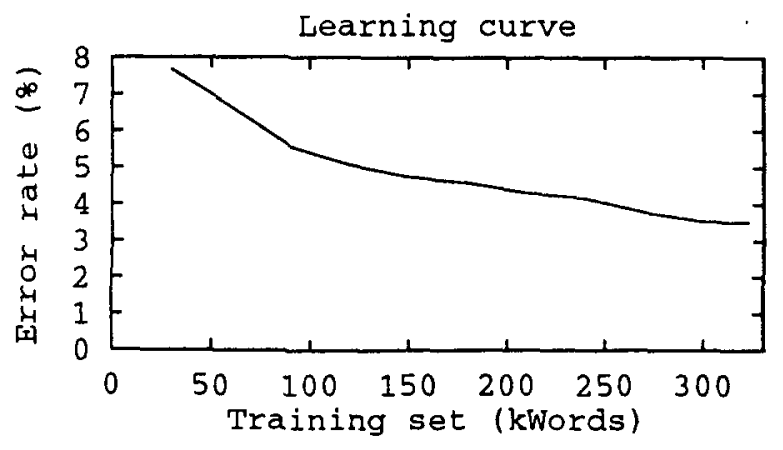

Figure 1: Learning curve for the statistical tagger on the Brown corpus.

\begin{tabular}{|c|c|c|c|}
\hline \multirow{2}{*}{$\begin{array}{c}\text { Ambiguity } \\
\text { (Tags/word) }\end{array}$} & \multicolumn{3}{|c|}{ Error rate $(\%)$} \\
\hline & $\begin{array}{l}\text { Statistical } \\
(\delta)\end{array}$ & $\begin{array}{l}\text { Tagger } \\
(\gamma)\end{array}$ & EngCG \\
\hline 1.000 & $4.72 \quad 4$ & 4.68 & \\
\hline 1.012 & & 4.20 & \\
\hline 1.025 & & 3.75 & \\
\hline 1.026 & & $3.72)$ & 0.43 \\
\hline 1.035 & & $3.48)$ & 0.29 \\
\hline 1.038 & & $3.40^{\prime}$ & \\
\hline 1.048 & & $3.20)$ & 0.15 \\
\hline 1.051 & & 3.14 & \\
\hline 1.059 & & $2.99)$ & 0.12 \\
\hline 1.065 & & 2.87 & \\
\hline 1.070 & & $2.80)$ & 0.10 \\
\hline 1.078 & & 2.69 & \\
\hline 1.093 & & 2.55 & \\
\hline
\end{tabular}

Table 1: Error-rate-ambiguity tradeoff for both taggers on the benchmark corpus. Parenthesized numbers are interpolated.

and partial disambiguation mode. Table 1 shows the error rate as a function of remaining ambiguity (tags/word) both for the statistical tagger, and for the EngCG-2 tagger. The error rate for full disambiguation using the $\delta$ variables is $4.72 \%$ and using the $\gamma$ variables is $4.68 \%$, both $\pm 0.18 \%$ with confidence degree $95 \%$. Note that the optimal tag sequence obtained using the $\gamma$ variables need not equal the optimal tag sequence obtained using the $\delta$ variables. In fact, the former sequence may be assigned zero probability by the HMM, namely if one of its state transitions has zero probability.

Previously unseen words account for $2.01 \%$, and lexical tag omissions for $0.15 \%$ of the total error rate. These two error sources are together exactly $1.00 \%$ higher on the benchmark corpus than on the Brown corpus, and account for almost the entire difference in error rate. They stem from using less complete lexical information sources, and are most likely the effect of a larger vocabulary overlap between the test and training portions of the Brown corpus than between the Brown and benchmark corpora.

The ratio between the error rates of the two taggers with the same amount of remaining ambiguity ranges from 8.6 at 1.026 tags/word to 28.0 at 1.070 tags/word. The error rate of the statistical tagger can be further decreased, at the price of increased remaining ambiguity, see Figure 2. In the limit of retaining all possible tags, the residual error rate is entirely due to lexical tag omissions, i.e., it is $0.15 \%$, with in average 14.24 tags per word. The reason that this figure is so high is that the unknown words, which comprise $10 \%$ of the corpus, are assigned all possible tags as they are backed off all the way to the root of the reverse-suffix tree.

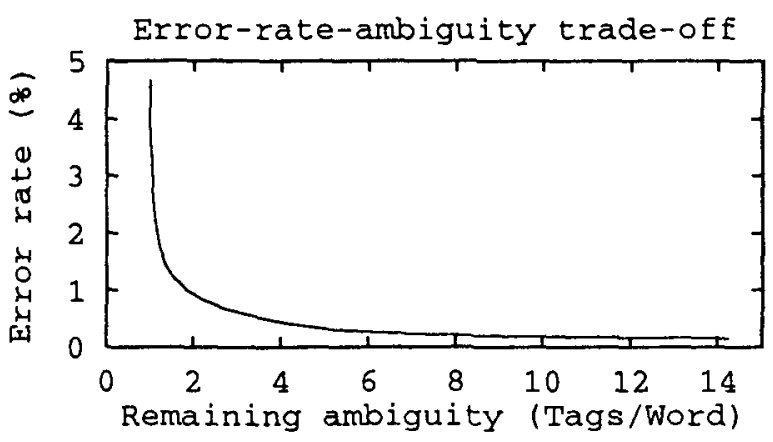

Figure 2: Error-rate-ambiguity tradeoff for the statistical tagger on the benchmark corpus.

\section{Discussion}

Recently voiced scepticisms concerning the superior EngCG tagging results boil down to the following:

- The reported results are due to the simplicity of the tag set employed by the EngCG system.

- The reported results are an effect of trading high ambiguity resolution for lower error rate.

- The results are an effect of so-called priming of the human annotators when preparing the test corpora, compromising the integrity of the experimental evaluations.

In the current article, these points of criticism were investigated. A state-of-the-art statistical tagger, capable of performing error-rate-ambiguity tradeoff, was trained on a 357.000-word portion of the Brown corpus reannotated with the EngCG tag set, and both taggers were evaluated using a separate 55,000 -word benchmark corpus new to both 
systems. This benchmark corpus was independently disambiguated by two linguists, without access to the results of the automatic taggers. The initial differences between the linguists' outputs $(0.7 \%$ of all words) were jointly examined by the linguists; practically all of them turned out to be clerical errors (rather than the product of genuine difference of opinion).

In the experiments, the performance of the EngCG-2 tagger was radically better than that of the statistical tagger: at ambiguity levels common to both systems, the error rate of the statistical tagger was 8.6 to 28 times higher than that of EngCG2. We conclude that neither the tag set used by EngCG-2, nor the error-rate-ambiguity tradeoff, nor any priming effects can possibly explain the observed difference in performance.

Instead we must conclude that the lexical and contextual information sources at the disposal of the EngCG system are superior. Investigating this empirically by granting the statistical tagger access to the same information sources as those available in the Constraint Grammar framework constitutes future work.

\section{Acknowledgements}

Though Voutilainen is the main author of the EngCG-2 tagger, the development of the system has benefited from several other contributions too. Fred Karlsson proposed the Constraint Grammar framework in the late 1980s. Juha Heikkilä and Timo Järvinen contributed with their work on English morphology and lexicon. Kimmo Koskenniemi wrote the software for morphological analysis. Pasi Tapanainen has written various implementations of the CG parser, including the recent CG-2 parser (Tapanainen 1996).

The quality of the investigation and presentation was boosted by a number of suggestions to improvements and (often sceptical) comments from numerous ACL reviewers and UPenn associates, in particular from Mark Liberman.

\section{References}

J-P Chanod and P. Tapanainen. 1995. Tagging French: comparing a statistical and a constraintbased method. In Procs. 7th Conference of the European Chapter of the Association for Computational Linguistics. pp. 149-15T, ACL, 1995.

K. W. Church. 1988. "A Stochastic Parts Program and Noun Phrase Parser for Unrestricted Text". In Procs. 2nd Conference on Applied Natural Language Processing, pp. 136-143, ACL, 1988.

K. Church. 1992. Current Practice in Part of Speech Tagging and Suggestions for the Future. In
Simmons (ed.), Sbornik praci: In Honor of Henry Kučera. Michigan Slavic Studies, 1992.

D. Cutting, J. Kupiec, J. Pedersen and P. Sibun. 1992. A Practical Part-of-Speech Tagger. In Procs. 3rd Conference on Applied Natural Language Processing, pp. 133-140, ACL, 1992.

S. J. DeRose. 1988. "Grammatical Category Disambiguation by Statistical Optimization". In Computational Linguistics 14(1), pp. 31-39, ACL, 1988.

N. W. Francis and H. Kučera. 1982, Frequency Analysis of English Usage, Houghton Mifflin, Boston, 1982.

R. Garside, G. Leech and G. Sampson (eds.). 1987. The Computational Analysis of English. London and New York: Longman, 1987.

B. Greene and G. Rubin. 1971. Automatic grammatical tagging of English. Brown University, Providence, 1971.

D. Hindle. 1989. Acquiring disambiguation rules from text. In Procs. 27th Annual Meeting of the Association for Computational Linguistics, pp. 118-125, ACL, 1989.

F. Jelinek and R. L. Mercer. 1980. "Interpolated Estimation of Markov Source Paramenters from Sparse Data". Pattern Recognition in Practice: 381-397. North Holland, 1980.

F. Karlsson. 1990. Constraint Grammar as a Framework for Parsing Running Text. In Procs. CoLing'90. In Procs. 14th International Conference on Computational Linguistics, ICCL, 1990.

F. Karlsson, A. Voutilainen, J. Heikkilä and A. Anttila (eds.). 1995. Constraint Grammar. A Language-Independent System for Parsing Unrestricted Text. Berlin and New York: Mouton de Gruyter, 1995.

B. Krenn and C. Samuelsson. The Linguist's Guide to Statistics. Version of April 23, 1996. http://coli.uni-sb.de/ christer.

C. G. de Marcken. 1990. "Parsing the LOB Corpus". In Procs. 28th Annual Meeting of the Association for Computational Linguistics. pp. 243251, ACL, 1990.

K. Oflazer and I. Kuruöz. 1994. Tagging and morphological disambiguation of Turkish text. In Procs. 4th Conference on Applied Natural Language Processing. ACL. 1994.

L. R. Rabiner. 1989. "A Tutorial on Hidden Markov Models and Selected Applications in Speech Recognition". In Readings in Speech Recognition: pp. 26i-296. Alex Waibel and KaiFu Lee (eds), Morgan Kaufmann, 1990.

G. Sampson. 1995. English for the Computer, Oxford University Press. 1995. 
C. Samuelsson. 1996. "Handling Sparse Data by Successive Abstraction". In Procs. 16th International Conference on Computational Linguistics, pp. 895-900, ICCL, 1996.

H. Schmid. 1994. Part-of-speech tagging with neural networks. In Procs. 15th International Conference on Computational Linguistics, pp. 172-176, ICCL, 1994.

P. Tapanainen. 1996. The Constraint Grammar Parser CG-2. Publ. 27, Dept. General Linguistics, University of Helsinki, 1996.

P. Tapanainen and A. Voutilainen. 1994. Tagging accurately - don't guess if you know. In Procs. 4 th Conference on Applied Natural Language Processing, ACL, 1994.

A. Voutilainen, 1995. "A syntax-based part of speech analyser". In Procs. 7th Conference of the European Chapter of the Association for Computational Linguistics, pp. 157-164, ACL, 1995.

A. Voutilainen and J. Heikkilä. 1994. An English constraint grammar (EngCG): a surface-syntactic parser of English. In Fries, Tottie and Schneider (eds.), Creating and using English language corpora, Rodopi, 1994.

A. Voutilainen, J. Heikkilä and A. Anttila. 1992. Constraint Grammar of English. A PerformanceOriented Introduction. Publ. 21, Dept. General Linguistics, University of Helsinki, 1992.

A. Voutilainen and T. Järvinen. "Specifying a shallow grammatical representation for parsing purposes". In Procs. 7th Conference of the European Chapter of the Association for Computational Linguistics, pp. 210-214, ACL, 1995. 


\section{Appendix: Reduced EngCG tag set}

\begin{tabular}{l}
\hline Punctuation tags: \\
\hline @colon \\
@comma \\
@dash \\
@dotdot \\
@dquote \\
@exclamation \\
@fullstop \\
@lparen \\
@rparen \\
@rparen \\
@rparen \\
@rparen \\
@lquote \\
@rquote \\
@slash \\
@newlines \\
@question \\
@semicolon \\
\hline Word tags: \\
\hline A-ABS \\
A-CMP \\
A-SUP \\
ABBR-GEN-SG/PL \\
ABBR-GEN-PL \\
ABBR-GEN-SG \\
ABBR-NOM-SG/PL \\
ABBR-NOM-PL \\
ABBR-NOM-SG \\
ADV-ABS \\
ADV-CMP \\
ADV-SUP \\
ADV-WH \\
BE-EN \\
\end{tabular}

BE-IMP
BE-INF
BE-ING
BE-PAST-BASE
BE-PAST-WAS
BE-PRES-AM
BE-PRES-ARE
BE-PRES-IS
BE-SUBJUNCTIVE
CC
CCX
CS
DET-SG/PL
DET-SG
DET-WH
DO-EN
DO-IMP
DO-INF
DO-ING
DO-PAST
DO-PRES-BASE
DO-PRES-SG3
DO-SUBJUNCTIVE
EN
HAVE-EN
HAVE-IMP
HAVE-INF
HAVE-ING
HAVE-PAST
HAVE-PRES-BASE
HAVE-PRES-SG3
HAVE-SUBJUNCTIVE
I
INFIARK

ING

N-GEN-SG/PL

N-GEN-PL

N-GEN-SG

N-NOM-SG/PL

N-NOM-PL

N-NOM-SG

NEG

NUM-CARD

NUM-FRA-PL

NUM-FRA-SG

NUM-ORD

PREP

PRON

PRON-ACC

PRON-CMP

PRON-DEM-PL

PRON-DEM-SG

PRON-GEN

PRON-INTERR

PRON-NOM-SG/PL

PRON-NOM-PL

PRON-NOM-SG

PRON-REL

PRON-SUP

PRON-IWH

V-AUXMOD

$\mathrm{V}$-IMP

V-INF

$V$-PAST

$V$-PRES-BASE

$\checkmark$-PRES-SG 1

V-PRES-SG2

V-PRES-SG3

$V$-SUBJUYCTIVE 